\title{
BMJ Open Maternal age at delivery and risk of allergy and asthma in the offspring: a systematic review and meta- analysis protocol
}

\author{
Yohannes Tesfaye Amera (D) , ${ }^{1}$ Abdoulie K Baldeh, ${ }^{1}$ Mohamed Mustafa Ali, ${ }^{1}$ \\ Emma Goksör, ${ }^{2}$ Göran Wennergren, ${ }^{3}$ Bright I Nwaru ${ }^{3}$
}

To cite: Amera YT, Baldeh AK, Ali MM, et al. Maternal age at delivery and risk of allergy and asthma in the offspring: a systematic review and metaanalysis protocol. BMJ Open 2020;10:e039288. doi:10.1136/ bmjopen-2020-039288

- Prepublication history and additional material for this paper is available online. To view these files, please visit the journal online (http://dx.doi.org/10. 1136/bmjopen-2020-039288).

Received 14 April 2020 Revised 31 August 2020 Accepted 25 September 2020

D) Check for updates

(c) Author(s) (or their employer(s)) 2020. Re-use permitted under CC BY-NC. No commercial re-use. See rights and permissions. Published by BMJ.

${ }^{1}$ School of Public Health and Community Medicine, Institute of Medicine, University of Gothenburg, Gothenburg, Sweden

${ }^{2}$ Department of Paediatrics, University of Gothenburg, Gothenburg, Sweden ${ }^{3}$ Krefting Research Centre, University of Gothenburg, Gothenburg, Sweden

Correspondence to Yohannes Tesfaye Amera; yoyetes20@gmail.com

\section{ABSTRACT}

Introduction While several perinatal factors have been linked to the risk of developing asthma and allergy in childhood, the role of maternal age at delivery remains uncertain. Some studies suggest that young maternal age at delivery may increase the risk, while other studies suggested a reduced risk. To provide a clearer appreciation of the underlying evidence, we plan to undertake a systematic review to synthesise previous studies that have investigated the association between maternal age at delivery and the risk of asthma and allergy in the offspring. Methods and analysis We will search PubMed, EMBASE, ISI Web of Science, Scopus and Google Scholar to identify relevant studies on the topic published in the databases from inception until October 2020. We will search databases of proceedings of international conferences, contact authors who have published on the topic and search the reference lists of the included studies in order to identify additional studies. Two investigators will independently screen the identified studies, perform data extraction and examine the risk of bias in the studies; a third investigator will arbitrate throughout these processes. We will use the Effective Public Health Practice Project tool for assessment of the risk of bias in included studies. We will perform random-effects meta-analysis to combine effect estimates from included studies judged to be homogeneous.

Ethics and dissemination Only data from the published literature will be included in this study, therefore no ethics approval is required. Our findings will be published in a peer-reviewed journal.

Protocol registration The protocol has been submitted for registration on PROSPERO, University of York, and Centre for Review and Dissemination, now awaiting the assignment of a registration number.

\section{BACKGROUND}

The risk of developing asthma and allergy in childhood may be established already in utero. ${ }^{1-4}$ The concept of fetal programming, that prenatal and intrauterine exposures surrounding the fetus may precipitate longlasting changes in the physiology and development of the fetus and subsequently later
Strengths and limitations of this study

- This is the first systematic review to address the association between maternal age at delivery and offspring asthma and allergy, hence the findings will contribute to growing knowledge on the role of prenatal and early life factors in the development of asthma and allergy in childhood.

- With a comprehensive search of the underlying literature with no language or geographical restriction, the findings from this review can be generalisable across different contexts.

- Outlining the review processes prior to undertaking the study ensures a high quality and reproducibility of our work.

- The definition of maternal age at delivery may differ across studies, thus may pose a challenge in combining data across studies.

- The current synthesis will provide the basis for designing mechanistic studies to understand the processes through which maternal age at delivery influences the development of asthma and allergy in the offspring.

risk of diseases, has been emphasised in the context of development of asthma and allergy in childhood. ${ }^{5-7}$ Although the specific mechanisms for the in utero development of asthma and allergy remain the subject of ongoing investigations, it is now generally accepted that the processes involve a combination of genetic and environmental factors. ${ }^{2-4}$

Over the past five decades, a range of perinatal factors have been investigated in relation to the development of asthma and allergy in childhood, including but not limited to diet, smoking, mode of delivery, use of medications (eg, antibiotics, proton pump inhibitors and acetaminophen) and indicators of psychosocial stress. ${ }^{89}$ Furthermore, the age of the mother at child's delivery, gestational weeks at delivery, the weight of the baby at delivery, as well as physiological development 
during infancy, have all also been linked to the development of asthma and allergy in childhood. ${ }^{10-16}$ The role of maternal age at delivery in the development of asthma and allergy in the offspring is however uncertain: while some studies have shown that child birth at a young age is a risk factor for the development of asthma and allergy in the offspring, other studies have reported that old age at delivery is a risk factor, yet some studies found no relationship between mother's age at birth and offspring's asthma and allergy. ${ }^{17-19}$ Although the specific mechanism through which maternal age at delivery influences the offspring's risk of developing asthma and allergy has not been elucidated, it has been suggested that this may involve a combination of hormonal, metabolic, immunological and genetic factors, which manifest during the fine-tuning of the processes of reproduction ${ }^{18}$ Clarifying the impact of maternal age at delivery on offspring's risk of developing asthma and allergy will contribute to ongoing evidence of the importance of reproductive events in later health of the child. Furthermore, determining an optimal age at child birth may contribute to preventive strategies for childhood asthma and allergy.

Given the conflicting findings from existing studies on the association between maternal age at delivery and risk of asthma and allergy in the offspring, there is therefore the need to synthesise the studies that have so far been published. This will provide a clearer appreciation of the underlying evidence base. The aim of this study is therefore to identify, critically appraise, and synthesise primary studies investigating the association between maternal age at delivery and the development of asthma and allergy in offspring. We hypothesise that younger maternal age may increase the risk of development of asthma and allergy in the offspring.

\section{METHOD}

This protocol is reported following the guidelines and recommendations of the Preferred Reporting Items for Systematic Reviews and Meta-Analyses (PRISMA-P). ${ }^{20}$

\section{Study types}

We will include all analytical observational epidemiological studies on the topic, which have been based on individual level data, including cohort, case-control and cross-sectional studies. We will exclude ecological studies, case reports, case series, reviews and animal studies.

\section{Study participants}

The study participants will include pregnant mothers and their offspring of any age.

\section{Types of exposure}

The exposure is mother's age at delivery. We will employ maternal age as defined in included studies, either age as a continuous variable or study-defined age categories.

\section{Outcome measures}

Our primary outcomes are self-reported or objectively defined asthma, atopic dermatitis/eczema, allergic rhinitis and food allergy. The secondary outcomes will include asthma exacerbations, the use of asthma medications, hospitalisation for asthma, wheeze and indicators of airway obstruction (forced expiratory volume in $1 \mathrm{~s}$, forced vital capacity, forced expiratory flow rate or alternative age appropriate pulmonary function tests (oscillometry or exhaled nitric oxide analysis)).

\section{Database searches to identify studies}

To identify relevant studies on the topic, we will search PubMed, EMBASE, ISI Web of Science, Scopus and Google Scholar. The databases will be searched for studies indexed from inception of respective databases until October 2020. Additional studies will be located by searching the references cited in studies identified from the database searches; from databases of the proceedings of international conferences, such as ISI Conference Proceedings Citation Index via Web of Knowledge, ZETOC (British Library); and by contacting authors who have previously published in the field.

\section{Search strategy}

With the help of librarians, we have developed a search strategy (online supplemental appendix 1) using the PubMed platform to identify and retrieve relevant studies. This search strategy will be adapted in searching the other databases. There will be no language restriction when searching the databases and we will translate papers published in languages other than English.

\section{Data management}

We will export all records retrieved from the databases to EndNote Library for de-duplication of publications. After de-duplication, the records will be exported to Rayyan reference management system, which will be used for study screening.

\section{Screening of literature}

Two investigators will independently screen the retrieved and de-duplicated records. In the first stage of the screening process, the title and abstracts of the retrieved records will be assessed for relevance. Following this, the full texts of potentially eligible papers will be independently assessed by the two reviewers. The screening process will be discussed by the two reviewers in order to resolve any discrepancies; any unresolved discrepancies between the two reviewers will be referred to a third reviewer for arbitration.

\section{Data extraction and management}

We will develop and customise a data extraction form to be used to collect relevant data from included studies. The data extraction form will be developed jointly by the study investigators and will be piloted before full use in the systematic review. Data extraction will be undertaken independently by two reviewers. Any disagreements during this process will be resolved through discussion and arbitration of any unresolved discrepancies by a third reviewer. 


\section{Data items}

As a minimum, we will summarise the following data items from each study: study author; country of conduct of study; study publication year; type of study design; study sample size; source of recruitment of study population; definition of maternal age at delivery and method of assessment; duration of follow-up (for follow-up studies); potential confounders included in studies; study outcomes and methods of assessment; analysis methods and key results.

\section{Quality assessment}

The methodological quality of the included studies will be assessed by two independent reviewers. Risk of bias assessment will be evaluated using the Effective Public Health Practice Project (EPHPP) tool. ${ }^{21}$ The EPHPP has six domains where assessment is made on various aspects of each study to evaluate the potential risk of bias, including study design, selection bias, confounding, blinding, study collection, withdrawals and dropouts. ${ }^{22}$ Based on the assessment and grading made across the six domains, we will derive a global quality grade for each study.

\section{Analysis}

We will construct tables to descriptively summarise the included studies. We will narratively synthesise the evidence from the studies and for studies judged to be clinically, methodologically and statistically reasonably homogeneous will be analysed using random-effects meta-analysis. The meta-analysis will be based on fully adjusted estimates from each included study. Heterogeneity between studies will be quantified using the $\mathrm{I}^{2}$ test. Where we have enough studies, possible reasons for heterogeneity between studies will be explored by performing subgroup analyses. Potential subgroups to be considered will include quality appraisal grading, study design and age of the child at the assessment of the outcomes. Furthermore, where feasible, we will undertake sensitivity analyses to evaluate the robustness of our findings and whether changing certain assumptions influences the overall conclusion of our results. For instance, by excluding all low-quality studies from the meta-analysis, we will evaluate whether the results obtained from highquality studies are different from results when all studies are combined; the same strategy will be used with regards to prospectively versus retrospectively designed studies, as well as studies conducted in low-income versus highincome countries.We will use the funnel plot and Egger's test to evaluate the potential for small-study effects. The meta-analysis will be undertaken using Stata statistical program V.16.

\section{Registration and reporting}

This protocol has been registered with the International Prospective Register of Systematic Reviews, now awaiting assignment of a registration number. The report of this review will be presented following the PRISMA and guidelines for Meta-analysis Of Observational Studies in Epidemiology. 2223

\section{Patient and public involvement}

Patient or the public were not involved in the development of the systematic review protocol and the study processes.

\section{DISCUSSION}

The emerging evidence linking maternal age at delivery to the development of asthma and allergy in the offspring is inconsistent, therefore, a systematic synthesis of published studies is essential to provide clarity, identify gaps and help to advance the field. This evidence synthesis will contribute to these goals. Although the mechanistic pathways through which maternal age at delivery influences the risk of asthma and allergy in the offspring have not been clearly elucidated, older maternal age is a marker of a number of factors already linked to the risk of asthma and allergy in the offspring, including increased gravidity, more children and decreased stressful life, all of which have been associated with a decreased risk of asthma and allergy in the offspring. ${ }^{24-27}$ Fluctuation in maternal oestradiol and oestrone levels are associated with ageing, ${ }^{28}$ and thus could provide potential pathways through which maternal age at delivery may influence offspring's asthma and allergy risk. Several studies have shown that maternal hormonal levels during pregnancy is associated with risk of asthma and allergy in the offspring. ${ }^{24-26} 29$ The definition of maternal age at delivery may differ across studies, thus may pose a challenge in combining data across studies. For example, some studies may use maternal age as a continuous variable, while others may divide maternal age into different groups; even among studies that divide maternal age into different groups, the age categories may differ between studies.

In conclusion, among several prenatal factors now identified to influence risk of asthma and allergy in the offspring, the role of maternal age at delivery remains uncertain, consequently an evidence synthesis of published studies on the topic is warranted. Through a comprehensive synthesis of the diverse literature on the topic, we will gain more clarity of the underlying evidence. This systematic review will therefore contribute to ongoing understanding of the impact of reproductive events in later disease risk in the offspring. Evidence generated from this systematic review may provide a motivation for undertaking mechanistic studies to uncover the underlying biological processes through which maternal age at delivery influences the risk of asthma and allergy in the offspring.

\section{Ethics and dissemination}

As the systematic review will be based on review of the published literature, no ethical permission is required. We will present our findings in international asthma and 
allergy conferences and will also be reported in a peerreviewed international journal in the field.

Contributors BIN conceived the study. YTA and AKB wrote the first draft of the protocol. BIN, EG, MMA and GW provided critical comments for improvement in all parts of the protocol. All authors read and approved the final manuscript submitted.

Funding The authors have not declared a specific grant for this research from any funding agency in the public, commercial or not-for-profit sectors.

Competing interests None declared.

Patient consent for publication Not required

Provenance and peer review Not commissioned; externally peer reviewed.

Supplemental material This content has been supplied by the author(s). It has not been vetted by BMJ Publishing Group Limited (BMJ) and may not have been peer-reviewed. Any opinions or recommendations discussed are solely those of the author(s) and are not endorsed by BMJ. BMJ disclaims all liability and responsibility arising from any reliance placed on the content. Where the content includes any translated material, BMJ does not warrant the accuracy and reliability of the translations (including but not limited to local regulations, clinical guidelines, terminology, drug names and drug dosages), and is not responsible for any error and/or omissions arising from translation and adaptation or otherwise.

Open access This is an open access article distributed in accordance with the Creative Commons Attribution Non Commercial (CC BY-NC 4.0) license, which permits others to distribute, remix, adapt, build upon this work non-commercially, and license their derivative works on different terms, provided the original work is properly cited, appropriate credit is given, any changes made indicated, and the use is non-commercial. See: http://creativecommons.org/licenses/by-nc/4.0/.

\section{ORCID iD}

Yohannes Tesfaye Amera http://orcid.org/0000-0003-2798-0267

\section{REFERENCES}

1 Duijts L. Fetal and infant origins of asthma. Eur J Epidemiol 2012;27:5-14.

2 Turner S. Perinatal programming of childhood asthma: early fetal size, growth trajectory during infancy, and childhood asthma outcomes. Clin Dev Immunol 2012;2012:1-9.

3 Tedner SG, Örtqvist AK, Almqvist C. Fetal growth and risk of childhood asthma and allergic disease. Clin Exp Allergy 2012;42:1430-47.

4 Grieger JA, Clifton VL, Tuck AR, et al. In utero programming of allergic susceptibility. Int Arch Allergy Immunol 2016;169:80-92.

5 Kwon EJ, Kim YJ. What is fetal programming?: a lifetime health is under the control of in utero health. Obstet Gynecol Sci 2017;60:506-19.

6 Barker DJP, Eriksson JG, Forsén T, et al. Fetal origins of adult disease: strength of effects and biological basis. Int J Epidemiol 2002;31:1235-9.

7 Barker DJ. In utero programming of chronic disease. Clin Sci 1998;95:115-28.

8 Rosas-Salazar C, Hartert TV. Prenatal exposures and the development of childhood wheezing illnesses. Curr Opin Allergy Clin Immunol 2017;17:110-5.

9 Wen H-J, Chiang T-L, Lin S-J, et al. Predicting risk for childhood asthma by pre-pregnancy, perinatal, and postnatal factors. Pediatr Allergy Immunol 2015;26:272-9.
10 Kloepfer KM. Maternal dietary antioxidant intake in pregnancy and childhood respiratory and atopic outcomes: birth cohort study. Pediatrics 2019;144:S8-9.

11 Yitshak-Sade M, Gorodischer R, Aviram M, et al. Prenatal exposure to $\mathrm{H} 2$ blockers and to proton pump inhibitors and asthma development in offspring. J Clin Pharmacol 2016;56:116-23.

12 Flanigan C, Sheikh A, Nwaru BI. Prenatal maternal psychosocial stress and risk of asthma and allergy in their offspring: protocol for a systematic review and meta-analysis. NPJ Prim Care Respir Med 2016;26:16021

13 Douros K, Moustaki M, Tsabouri S, et al. Prenatal maternal stress and the risk of asthma in children. Front Pediatr 2017;5:202.

14 Silvestri M, Franchi S, Pistorio A, et al. Smoke exposure, wheezing, and asthma development: a systematic review and meta-analysis in unselected birth cohorts. Pediatr Pulmonol 2015;50:353-62.

15 Mitselou N, Hallberg J, Stephansson O, et al. Cesarean delivery, preterm birth, and risk of food allergy: nationwide Swedish cohort study of more than 1 million children. J Allergy Clin Immunol 2018;142:1510-4.

16 Sordillo JE, Scirica CV, Rifas-Shiman SL, et al. Prenatal and infant exposure to acetaminophen and ibuprofen and the risk for wheeze and asthma in children. J Allergy Clin Immunol 2015;135:441-8.

17 Abid Z, Oh SS, Hu D, et al. Maternal age and asthma in Latino populations. Clin Exp Allergy 2016;46:1398-406.

18 Gómez Real F, Burgess JA, Villani S, et al. Maternal age at delivery, lung function and asthma in offspring: a population-based survey. Eur Respir J 2018;51:1601611.

19 Laerum BN, Svanes C, Wentzel-Larsen T, et al. Young maternal age at delivery is associated with asthma in adult offspring. Respir Med 2007;101:1431-8.

20 Moher D, Shamseer L, Clarke M, et al. Preferred reporting items for systematic review and meta-analysis protocols (PRISMA-P) 2015 statement. Syst Rev 2015;4:1.

21 EPHPP P. Quality assessment tool for quantitative studies. Hamilton: the effective public health practice project. 20, 2010. http://www. ephpp. ca/PDF/Quality\% 20Assessment

22 McLeroy KR, Northridge ME, Balcazar $\mathrm{H}$, et al. Reporting guidelines and the American Journal of public health's adoption of preferred reporting items for systematic reviews and meta-analyses. Am J Public Health 2012;102:780-4.

23 Fleming PS, Koletsi D, Pandis N. Blinded by PRISMA: are systematic reviewers focusing on PRISMA and ignoring other guidelines? PLOS One 2014;9:e96407.

24 Flanigan C, Sheikh A, DunnGalvin A, et al. Prenatal maternal psychosocial stress and offspring's asthma and allergic disease: a systematic review and meta-analysis. Clin Exp Allergy 2018;48:403-14.

25 Fan T, Hu Y, Xin J, et al. Analyzing the genes and pathways related to major depressive disorder via a systems biology approach. Brain Behav 2020;10:e01502..

26 Grunewald S, Jank A. New systemic agents in dermatology with respect to fertility, pregnancy, and lactation. J Dtsch Dermatol Ges 2015;13:277-90.

27 Baïz N, Just J, Chastang J, et al. Maternal diet before and during pregnancy and risk of asthma and allergic rhinitis in children. Allergy Asthma Clin Immunol 2019;15:40.

28 Dorgan JF, Reichman ME, Judd JT, et al. Relationships of age and reproductive characteristics with plasma estrogens and androgens in premenopausal women. Cancer Epidemiol Biomarkers Prev 1995;4:381-6.

29 Pali-Schöll I, Motala C, Jensen-Jarolim E. Asthma and allergic diseases in pregnancy a review. World Allergy Organ $J$ 2009;2:26-36. 